\title{
Electron Microscopy of the SARS Coronavirus
}

\author{
D.R. Beniac, S.L. deVarennes, A. Andonov, T.F. Booth
}

National Microbiology Laboratory, 1015 Arlington Street, Winnipeg, Manitoba, R3E 3R2, Canada.

The outbreak of atypical pneumonia referred to as severe acute respiratory syndrome (SARS) affected several countries during the 2002-2003 winter flu season. The causative agent was named publicly by the world health organization as the "SARS coronavirus" (SARS-CoV) on 16 April 2003, and the virus was sequenced by May 2003 by two independent groups [1,2]. The SARS-CoV RNA genome encodes several structural proteins including; the spike glycoprotein (S), membrane glycoprotein (M), small envelope protein (E), and the nucleocapsid phosphoprotein (N). The host cell-surface molecule angiotensin-converting enzyme 2 (ACE2) is the receptor for the SARS-CoV spike protein [3].

We have grown the SARS-CoV in Vero E6 cells in BSL3 containment. The virus was purified by iodoxanol gradient centrifugation, and inactivated by gamma-irradiation. We then imaged the SARS-CoV in a FEI Tecnai 20 TEM at $200 \mathrm{kV}$. In this investigation we stained SARS-CoV with methylamine tungstate (A), and also imaged it unstained frozen hydrated (B, C). In addition we incubated SARS-CoV with soluble ACE2, or neutralizing antibodies, and immuno-labelled both preparations with $10 \mathrm{~nm}$ gold (D, F; respectively). For the cryo-EM image analysis investigation two data sets were processed. These contained either individual spike images of SARS-CoV, or SARS-CoV incubated with soluble ACE2. These data sets were then processed by image analysis procedures to generate $3 \mathrm{D}$ reconstructions of both the spike, and the spike complexed with ACE2 $[4,5]$. A notable feature of the SARS-CoV spike is its large mass $\sim 500 \mathrm{kDa} /$ trimer, it has a striking club shape, and appears like a three bladed propeller from the end-on perspective (E). The spike protrudes $16 \mathrm{~nm}$ above the viral envelope. The precise location of ACE2 binding on the distal end of the spike is centered at $7 \mathrm{~nm}$ from the central axis of the spike, with a $3 \mathrm{~nm}$ gap between the axis of symmetry and ACE2. One ACE2 molecule was bound to each of the three propeller-like blades of the spike, making a structure $22 \mathrm{~nm}$ high.

One of the challenges that face a diagnostic virology EM laboratory is rapid detection, structural preservation, and the appropriate fixation or inactivation for BSL2 and BSL3 pathogens. These initial rapid detection procedures are best achieved by negative staining, and can then be followed by subsequent investigations using alternative techniques which can include cryo-electron microscopy, electron tomography, and image analysis procedures. Particularly challenging are the enveloped viruses, that are sensitive to both mechanical and chemical conditions used in their purification and inactivation.

\section{References}

[1] M.A. Marra et al., Science. 300, 1399-1404, (2003).

[2] P.A. Rota et al., Science. 300, 1394-1399, (2003).

[3] W. Li et al., Nature 426, 450-454 (2003).

[4] D.R. Beniac et al., Nat. Str. Mol. Biol. 13, 751-752, (2006)

[5] D.R. Beniac et al., PLoS One. 2(10): e1082. doi:10.1371/journal.pone.0001082, 1-7, (2007). 


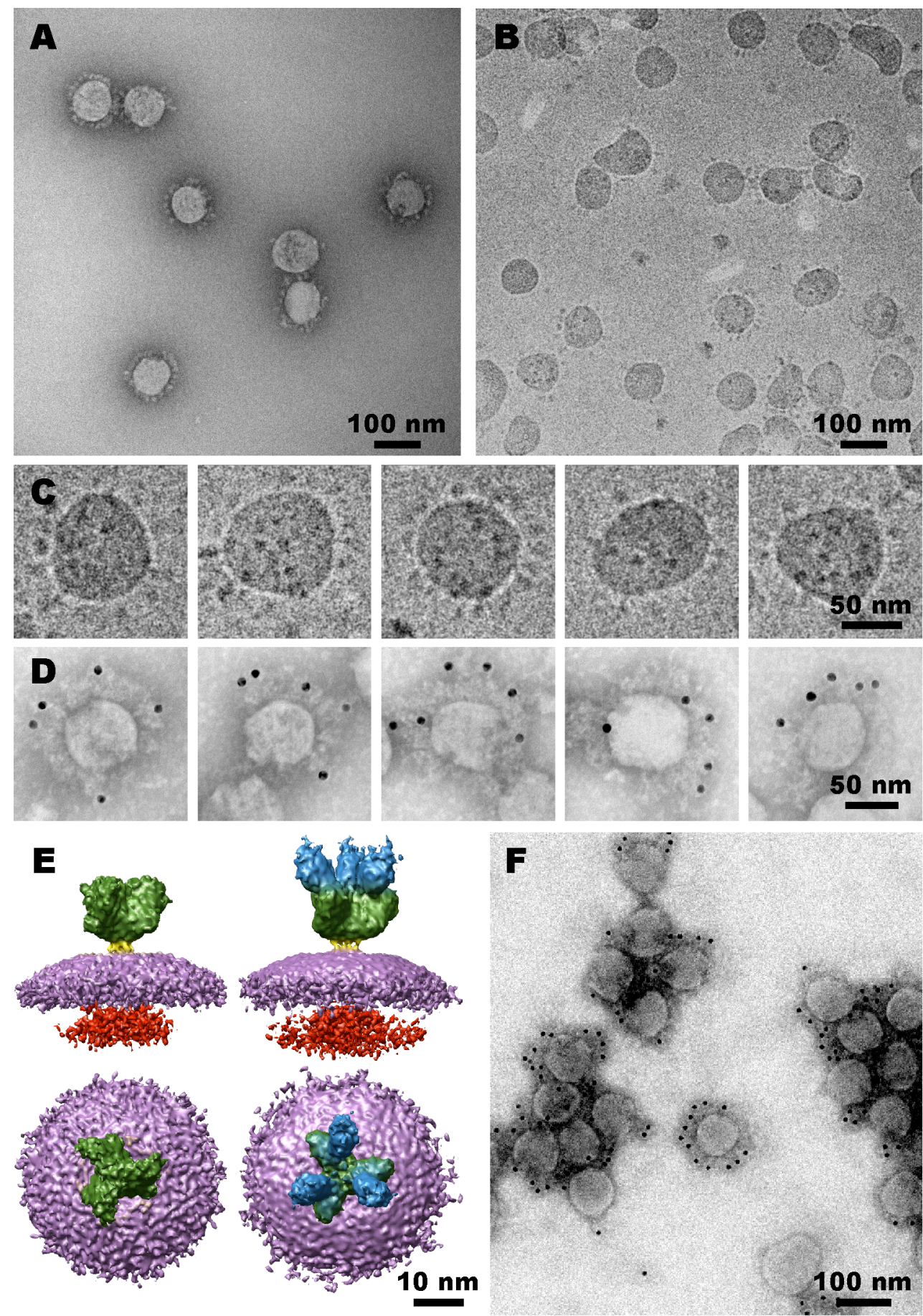

Images of the SARS coronavirus: (A) SARS-CoV stained with methylamine tungstate. (B) Gamma irradiated SARS-CoV imaged frozen hydrated. (C) Gallery of individual frozen hydrated SARS-CoV. (D) Immuno-EM of SARS-CoV incubated with soluble ACE2, and $10 \mathrm{~nm}$ gold. (E) $3 \mathrm{D}$ reconstructions of the SARS-CoV spike with, and without its receptor ACE2, shown from the side and end-on perspectives (top, bottom). Color scheme: ACE2, blue; spike, green; stalk, yellow; envelope, purple; nucleocapsid, red. (F) Immuno-EM of SARS-CoV using a neutralizing monoclonal antibody, and $10 \mathrm{~nm}$ gold. 